\title{
Improvement of the Gaussian Mixture Model Based on EmguCV Motion Target Detection Design
}

\author{
Qingyu Guo ${ }^{1, a^{*}}$ and Zheng Zhang ${ }^{1, b}$ \\ Zhongyuan University of Technology, Zhengzhou, 450007, China \\ aguo_qing_yu@yahoo.com, b zhang_zhengfly@163.com
}

\begin{abstract}
Keywords: K-means; Improved frame difference; Moving targets detection; EmguCV; The Gaussian mixture model
\end{abstract}

\begin{abstract}
Introduces a kind of video moving object detection based on mixture Gaussian model design, mainly combined with frame difference method and the Gaussian mixture model, then use K-means clustering algorithm, for complex scenarios different update rate is realized by using different background region, to complete the moving target detection, respectively, compared to traditional Gaussian mixture model (Gaussian mixture model, GMM) and the traditional frame difference detection method, in order to better solve the problem of motion target detection in complex scene, and mainly analyze based on frame difference and EmguCV framework, and finally using C\# and EmguCV framework for moving targets detection. Application through the experiment show that the design is easy to use, and the performance is better.
\end{abstract}

\section{Introduction}

Video motion object detection technology is to split from the image of the video sequence which obtained from the monitoring equipment, divided into background and moving object and extract the moving object information, on the basis of general interest in moving objects segmented from the background [1]. This technology is widely applied in video monitoring, artificial intelligence, aerospace, urban traffic, and other fields. Classical detection methods are mainly frame difference method[2], [3-4], background subtraction, division and optical flow method[5], etc., among them, the background subtraction division principle is to the current frame and background image difference, the motion target area, has simple operation, the characteristics of high precision become the commonly used algorithm is widely used. But the background subtraction division are greatly influenced by light, weather and other external conditions, how to establish the background image, becomes the key to background subtraction division effect is good or bad.

EmguCV [6] is the .Net version of the OpenCV package library, in the .Net compatible programming language such as $\mathrm{C \#}, \mathrm{VB}, \mathrm{VC}++$ and IornPython can directly call the image processing method, can encapsulate library on Windows, Linux, iPhone, Android, and many other platforms, it not only inherited the OpenCV all performance and characteristics, and in supporting the cross-platform operation, and contains image class based on color and depth; Can automatic memory management and garbage collection; XML sequence images; The XML document and intelligent support; Choose to use the image class or direct call OpenCV function; The basic operation of image pixels[7], etc.

\section{Background Acquisition}

The Traditional Gaussian Mixture Model Definition. In order to obtain ideal background, is more commonly used by C.stauffer modeling[8], the Gaussian mixture model proposed by using multiple Gaussian function at each pixel location modeling, using the pixel iteration parameters update, adapt to the background, illumination changes, and so on and so forth.

Traditional Gaussian mixture model (Gaussian mixture model, GMM) of each pixel in the image is principle to set up $K$ independent Gaussian models ( $K$ generally between 3 and 5 ), for the $t$ 
moment pixels of sample values $I_{t}(x, y)$, its probability density function of the $K$ multidimensional Gaussian distribution function by a weighted and to represent the probability density function:

$$
P\left(I_{t}(x, y)\right)=\sum_{i=1}^{K} \omega_{i, t} \eta_{i, t}\left(I_{t}(x, y), \mu_{i, t}, \sum_{i, t}\right)
$$

Among them, $K$ is the number of Gaussian component, $\omega_{i, t}$ is the $i$ weight of a Gaussian components ( $\sum_{i=1}^{K} \omega_{i, t}=1$, the summation weight of $\boldsymbol{i}$ should be 1 ); $\boldsymbol{\mu}_{i, t}$ is a Gaussian weight average of the $\boldsymbol{i} ; \sum_{i, t}$ is the $\boldsymbol{i}$ Gaussian component of covariance, $\eta_{i, t}\left(I_{t}(x, y), \mu_{i, t}, \sum_{i, t}\right)$ is the $t$ time $\boldsymbol{i}$ Gaussian probability density function, Gaussian distribution is defined as:

$$
\eta_{i, t}\left(I_{t}(x, y), \mu_{i, t}, \sum_{i, t}\right)=\frac{1}{(2 \pi)^{\frac{n}{2}}\left|\sum_{i, t}\right|^{\frac{1}{2}}} \times e^{-\frac{1}{2}\left(I_{t}(x, y)-\mu_{i, t}\right)^{T} \sum_{i, t}^{-1}\left(I_{t}(x, y)-\mu_{i, t}\right)}
$$

Among them, $n$ is the dimension of sample values $I_{t}(x, y)$.

Parameter Updating and Background Matching. Modeling is the Gaussian mixture model in every place, in turn, matching the current frame multiple pixels of each Gaussian component, select one or several modeling as a background model, other are the prospects of the model. If the currently selected value meet the conditions, the match is successful, the decision that point as the background, or for the future.

General situation, the choice of background modeling method is as follows:

(1) To all $K$ for the Gaussian component in the Eq. 1 according to the weight and the ratio of the square of the standard deviation ( $\omega_{i} / \sigma_{k}^{2}$ ) from big to small arrangement;

(2) Select the first $B$ as the background model, and satisfy the relationship:

$$
B=\arg \min _{b}\left(\sum_{i=1}^{K} \omega_{i, t}>T_{w}\right)
$$

Among them, $T_{w}$ is for the selected background, the probabilities of pixel values remain the same as Gaussian component weights selected threshold.

When a new frame for needs and has been $K$ ranked Gaussian component matching, the matching condition is:

$$
\left|x_{t}-\mu_{i, t-1}\right|<2.5\left(x_{t}, \mu_{i, t},\right)
$$

Meet the conditions of Eq. 41 of a Gaussian component, classified as the background model, and will conform to the existing Gaussian distribution point to parameter updating background, and the parameters according to the following update:

$$
\begin{aligned}
& \mu_{i, t}=(1-\alpha) \mu_{i, t-1}+\alpha x_{t} \\
& \sigma_{i, t}=(1-\beta) \sigma_{i, t-1}+\beta\left(x_{t}-\mu_{i, t-1}\right)^{2} \\
& \beta=\frac{\alpha}{\omega_{i, t}}
\end{aligned}
$$

Other Gaussian components remain the same. Update weights updated according to the following formula:

$$
\omega_{i, t}{ }^{\prime}=\omega_{i, t-1}+\lambda r
$$


$\alpha, \beta, r$ are all is updated coefficient, and when $\lambda=1$, the pixel values matching with Gaussian distribution, $\lambda=0$ does not match.

When the $K$ Gaussian distribution are not matching with the current pixel, argues that pixel points for future, needed to recreate a Gaussian model instead of the priority of minimum gauss distribution, a Gaussian distribution for the new set up the larger initial variance value, the average for the current pixel values.

The new model has a weight of:

$$
\omega_{i, t}=(1-r) \omega_{i, t-1}
$$

Finally, the normalization of all the weight coefficient of:

$$
\omega_{i, t}=\frac{\omega_{i, t}^{\prime}}{\sum_{i=1}^{K} \omega_{i, t}^{\prime}}
$$

For each frame of video sequence images, after modeling the Gaussian mixture model is used to collect the target background, then using background subtraction division for moving targets.

\section{Traditional Algorithm and Improved}

Three Frame Difference Algorithm. In the process of image processing, the frame difference method is by calculating the difference in value between two adjacent frames to get moving target. Two frames difference method can simply and quickly obtain the target movement area, but the difference only keep relative changes of information, when the target's movement speed too fast, it's easy to have a double and cavitation. Three frame difference method is based on two frames difference method, an improved two frame difference method can effectively overcome the phenomenon of double. This article adopts the method of three consecutive frames difference, the adjustment of dynamic threshold, the extracting an outline of the clear objectives, and shall not affect the processing speed, its principle is as follows:

$$
\begin{aligned}
& d_{1 k}= \begin{cases}1 & \left|P_{i}(x, y)-P_{i+1}(x, y)\right|>T+\Delta T_{1} \\
0 & \left|P_{i}(x, y)-P_{i+1}(x, y)\right| \leq T+\Delta T_{1}\end{cases} \\
& d_{2 k}= \begin{cases}1 & \left|P_{i+1}(x, y)-P_{i+2}(x, y)\right|>T+\Delta T_{2} \\
0 & \left|P_{i+1}(x, y)-P_{i+2}(x, y)\right| \leq T+\Delta T_{2}\end{cases} \\
& \Delta T_{1}=\lambda \frac{1}{M \times N} \sum_{x=0}^{M-1} \sum_{y=0}^{N-1}\left|P_{i}(x, y)-P_{i+1}(x, y)\right| \\
& \Delta T_{2}=\lambda \frac{1}{M \times N} \sum_{x=0}^{M-1} \sum_{y=0}^{N-1}\left|P_{i+1}(x, y)-P_{i+2}(x, y)\right|
\end{aligned}
$$

Among them, $d_{1 k}$ and $d_{2 k}$ are for the difference image, $\lambda$ is restrain coefficient, can undertake adjustment according to actual needs, this algorithm use the value of 2; The resolution of the images for each frame, and its value of pixels detection area; $T$ as the between-cluster variance method to determine the effect of the target and the background; $\Delta T_{1}$ and $\Delta T_{2}$ are the dynamic threshold, the changes reflect the light in the process of target detection, the light changes, the more obvious, the greater its value. Finally, the image and the logic operation, get the outline of moving targets, so as to determine the goals of the movement.

$$
\begin{aligned}
& d_{i}=d_{1 i} \cap d_{2 i} \\
& D_{i}= \begin{cases}1 & \mathrm{~d}_{i}=1 \\
0 & \mathrm{~d}_{i}=0\end{cases}
\end{aligned}
$$


Among them, $D_{i}=1$ said prospects, $D_{i}=0$ said background points.

Improve Algorithm. Through the study found that the traditional Gaussian mixture model is not very good full of moving targets detection area, especially in complex scenes, high sensitivity to noise, which influence the accurate detection of target; In addition, when moving targets by static mutate into movement, illumination, and branches swaying complex scenario, the traditional Gaussian mixture model can't good to remove the interference of noise. In this paper, the traditional Gaussian mixture model was improved, to solve the above problems.

In order to eliminate interference factors, when the extracted pixel does not conform to the background, can be recorded by method of counting pixels is not in conformity with the background of The Times, when the pixel does not belong to establish the background image and pixel values change slowly, judgment that point to the noise, and makes the counter plus one, otherwise the reset.

$$
\text { count }= \begin{cases}\text { count }+1 & I_{i}(x, y) \notin B_{i}(x, y) \cap\left(I_{i}(x, y)-I_{i-1}(x, y)\right)<T_{2} \\ 0 & \text { other }\end{cases}
$$

Among them, the count for the counter, $\mathrm{I}_{i}(x, y)$ and $B_{i}(x, y)$ at a certain moment, respectively and background values of pixels, $T_{2}$ as the threshold. When the count reaches the threshold set, to update the background.

\section{Design Implementation}

Algorithm in this paper the hardware test platform for $2.5 \mathrm{GHz}$ CPU, 4 g RAM, Windows 7 32-bit operating system, the PC software platform for Visual Studio 2010, using C\# and EmguCV framework development. In testing, in the process of target in image sequence is preprocessed first, by modeling the Gaussian mixture model for target background, using background subtraction division for moving targets. Reuse K-means clustering algorithm, for complex scenes using different background region to realize different update rate, complete the moving target detection, get complete moving targets. The algorithm flow chart is shown in Fig. 1.

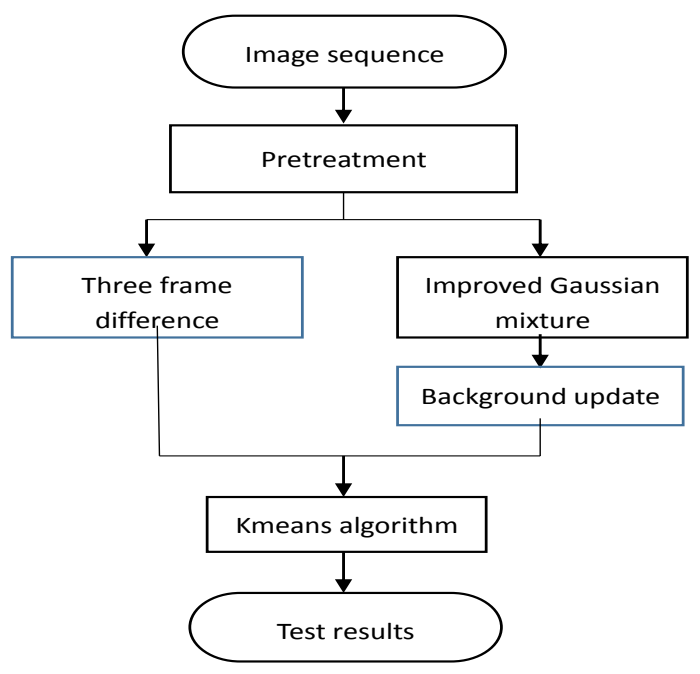

Figure 1. Algorithm flow chart

Fig. 2 is under the condition of the traditional algorithm, the detection of 139 frames in video sequences and the 140th frame of motion target detection result. Fig. 2(c) is the second frame difference method with the Gaussian model of moving target detection is binarization image. Fig. 3 is the result of the algorithm in this paper to get by the experimental results it can be seen that in the high-speed moving target movement, the traditional algorithm can detect moving targets; however, the detection of the target has a lot of interference factors. In this paper, using three frame difference and improve the operation of the Gaussian mixture model and object in high speed movement, can detect the moving targets. 


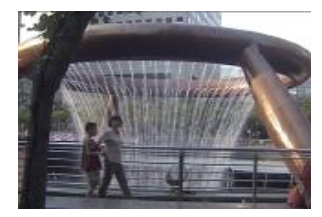

The 139 Frame (a)

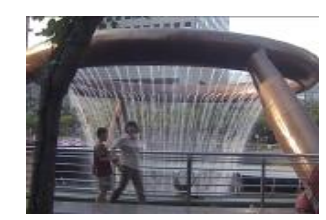

The 140 Frame (b)

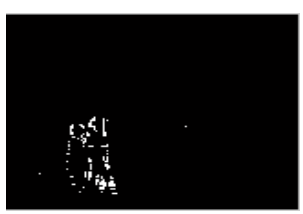

Test results (c)

Figure 2. Traditional methods

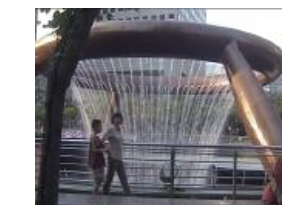

The 139 Frame (a)

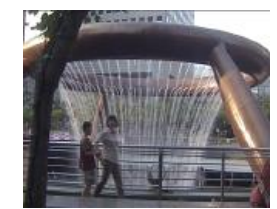

The 140 Frame (b)

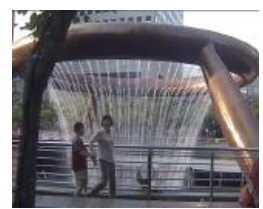

The 141 Frame (c)

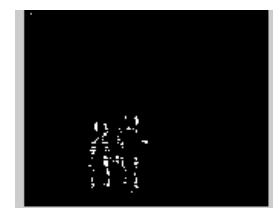

Test results $(\mathrm{d})$

Figure 3. Algorithm to deal with the results in this paper

\section{Conclusion}

Designed in this paper, based on EmguCV improvement of moving target detection system, the Gaussian mixture model than regular motion target detection system has stronger ability of image processing and intelligent processing, realized the detection of moving targets. Innovation point of this article is a combination of Gaussian mixture model is put forward and the algorithm of three frame difference method both advantages, can be adapted to the changing scenes, and overcome the inter-frame difference to extract the target is not accurate, easy to generate "double" and the influence of the hole, and easier to implement by a shadow suppression algorithm, effectively suppresses the existence of the shadow, at last, by using K-means clustering algorithm, for complex scenarios different update rate is realized by using different background region, to extract the moving object effectively. The experimental results show that the algorithm is not sensitive to the light background disturbance, have certain robustness and real-time, algorithm is fast and easy to implement, in the smart security, traffic real-time monitoring has extensive applicability, and have good economic benefit.

\section{References}

[1] X.F. Wei, X. Liu: Laser technology, Vol. 4 (2013) No.4, p.519

[2] L.X. Xue, Y.L. Luo and Z.C. Wang: Application Research of Computers, Vol. 28 (2011) No.4, p.1551

[3] N.N. He, J.P. Du: Journal of Beijing Technology and Business University (Natural Science Edition), Vol. 24 (2009) No.4, p.34

[4] Y. Wang, Y.J. Cao, F.F. Lang, H. Huang and P.P Yu: Journal of Electronic Science and technology, Vol. 24 (2012) No.4, p.31

[5] W. Wei and Q. Wu: Computer Engineering and Design, Vol. 35 (2014) No.3, p.949

[6] Y.L. Hua and W.J. Liu: Journal of Computer Applications, Vol. 34 (2014) No.2, p.580

[7] N. Xu: Journal of electronic technology in ShanXi, (2015), p.41

[8] B. Lu, L.L. Wang and D. Pei: Laser \& Infrared, (2016) No.2, p.240

[9] M. Zhu: Computer Knowledge and Technology, Vol. 11 (2015) No.28, p.141

[10] W. Guo, X.Y. Liu and Z.J. Xiao: Computer Engineering and Applications , (2015) No.18, p.148 